\title{
The Research of Shadow Detection and Removal Algorithm
}

\author{
Jiande Fan ${ }^{1, a}$ and Yanliu Nie Ni, b $^{1}$ \\ ${ }^{1}$ Information Engineering Institute, Zhengzhou University of Industrial Technology, Xinzheng, \\ 451100, China \\ a568498042@qq.com, b361045705@qq.com
}

Keywords: Shadow detection; Moving object; Object recognition; Image matching

\begin{abstract}
Shadow detection and removal is a key problem in computer vison. Shadow can lead to loss or disruption of computer vision information, and then results in unstable situations or even failure of edge extraction, object recognition and image matching in image processing. The thesis concentrates on the research of moving object shadow detection and removal, and proposed the shadow detection and removal algorithm based on RGB and $\mathrm{YCbCr}$ color space. The experimental results show the algorithm based on the $\mathrm{YCbCr}$ color space have the lowly advantage.
\end{abstract}

\section{阴影检测与去除算法研究}

\author{
范建德 ${ }^{1, a}$, 聂燕柳 ${ }^{1, b}$ \\ 1.郑州工业应用技术学院 信息工程学院，中国 河南 新郑 451100 \\ a568498042@qq.com, b361045705@qq.com
}

摘要：阴影检测与去除是计算机视觉中的一项关键技术。阴影会对运动物体信息造成一定的 损失或干扰，这将导致计算机图形处理过程中将导致响应区域的边缘提取、目标识别以及特 征匹配等的结果不稳定甚至失败。论文围绕运动目标阴影检测与去除问题, 提出了基于 RGB 和 $\mathrm{YCbCr}$ 颜色空间的阴影检测和去除算法, 仿真结果表明：基于 $\mathrm{YCbCr}$ 颜色空间的算法在 运算量较低的同时，对阴影检测与去除有较强的鲁棒性。

关键词：阴影检测；运动目标；目标识别；特征匹配

\section{1. 引言}

阴影对人类来说是一种司空见惯的现象，它具有和目标物体几乎一样的行动、运动特征、轮 廓, 由于阴影的存在可能引起物体的错误分类或者导致不同物体的相互融合, 从而改变检测 到的物体的形状和大小, 严重影响图像的各种精细处理工作, 如图像的精确边缘检测、物体 检测、场景分析和图像特征的匹配等[1-3]。因此，场景中物体阴影的有效监测和去除是非常 重要的, 如果对图像、视频进行视觉处理之前就能预先检测并去除阴影, 就可以创造出更逼 真的场景、更高效的压缩算法，使得计算机视觉会得到更广泛的应用。阴影种类繁多，有基 于几何特性的 [4-5], 有基于阴影灰度性质 [6]（如阴影边缘灰度渐变、突变，阴影分布是否均 匀）的，还有基于阴影形成原因的，但目前没有一种通用的阴影去除方法能对所有的阴影有 理想的处理效果 ${ }^{[7]}$ 。

\section{2. 归一化图像RGB的算法}

基于归一化 RGB 的阴影检测与去除算法是一种简单而有效的阴影处理方法。令 $\mathrm{R}, \mathrm{G}, \mathrm{B}$ 为 RGB 图像中某个像素的红色、绿色和蓝色, 它们的数值是 0 到 1 之间的实数; 该方法首先对 RGB 图像进行归一化处理: 


$$
\operatorname{norm\mathrm {R}}=\frac{r}{r+g+b}
$$

$$
n o r m \mathrm{G}=\frac{g}{r+g+b}
$$

$$
n o r m \mathrm{~B}=\frac{b}{r+g+b}
$$

通过这种计算方法, 可以将图像中的红黄蓝信息归一化, 将亮度信息从亮度色度饱和度中分 离出来, 从而避免了亮度变化对场景中目标的影响。如下所示:

$$
S P_{R G B}=\left\{\begin{array}{c}
1, \quad \text { abs }\left(\text { norm } \mathrm{R}_{f g}-\text { norm } \mathrm{R}_{b g}\right) \leq \varepsilon \wedge \\
a b s\left(\text { norm } \mathrm{G}_{f g}-\text { norm } \mathrm{G}_{b g}\right) \leq \varphi \wedge \\
a b s\left(\text { norm } \mathrm{B}_{f g}-\text { norm } \mathrm{B}_{b g}\right) \leq \gamma ; \\
0, \quad \text { else. }
\end{array}\right.
$$

其中, $f g$ 代表输入的当前图像, $b g$ 代表通过背景建模得到的背景图像。图 1 为交通视频监 控图像基于归一化 RGB 的阴影检测结果以及各个通道 (nor $m \mathrm{R}$, normG, nor h) 图像, $\varepsilon 、 \varphi 、 \gamma$ 的具体取值需要根据实验进行确定。

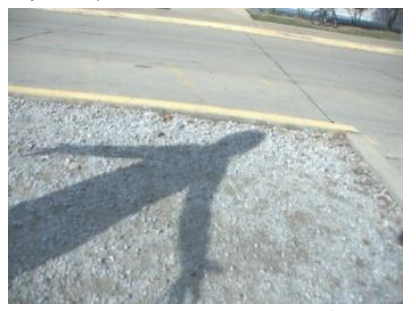

(a)阴影图像

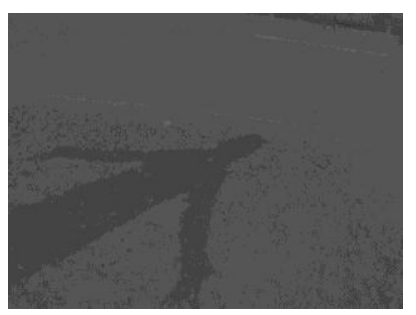

(d)阴影图像 normR 通道

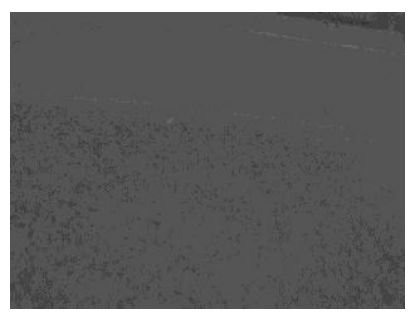

(g)背景图像 normR 通道

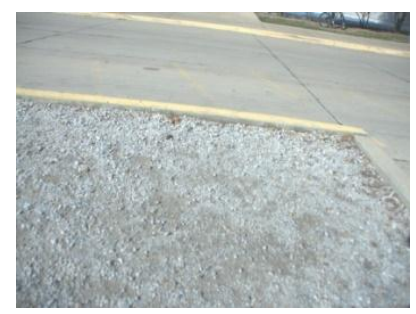

(b)背景图像

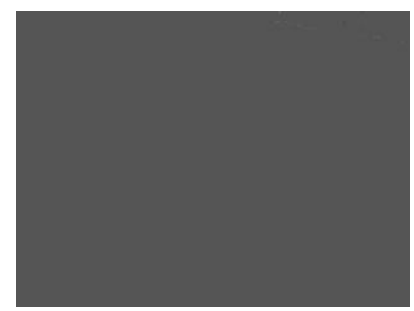

(e)阴影图像 normG 通道

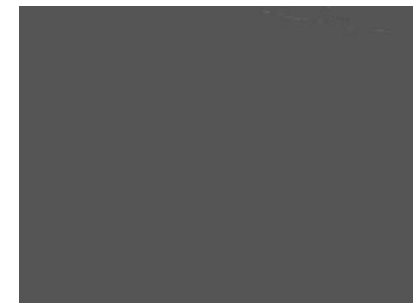

(h)背景图像 normG 通道

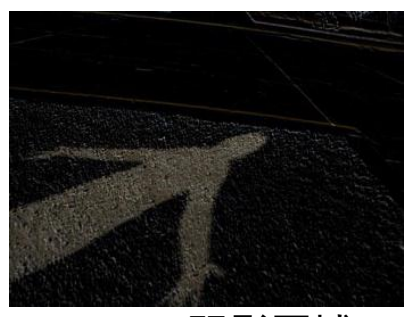

(c)阴影区域

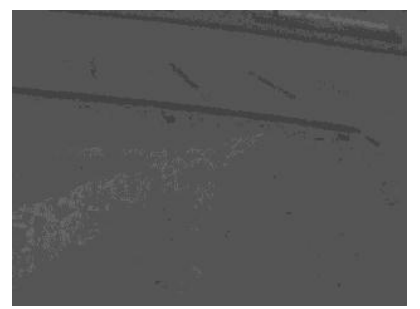

(f)阴影图像 normB 通道

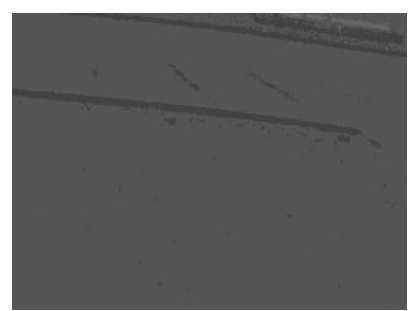

(i)背景图像 normB 通道

图 1 基于归一化 RGB 的阴影检测

基于归一化的阴影处理算法计算简单，效果较好，但是缺点也比较明显：在场景中的目标物 体是亮度较高的时候, 会出现目标漏检的情况。所以这种算法在阴影检测与去除方面实用性 不高, 局限性较大。

\section{3. 基于YCbCr的阴影检测与去除}


YCbCr 是一种视频格式, 主要用于优化彩色视频信号的传输, 使其向后相容老式黑白电视。 $\mathrm{YCbCr}$ 是 $\mathrm{YUV}$ 的子集, $\mathrm{Y}$ 是指图像中的亮度分量, $\mathrm{Cb}$ 指蓝色色度分量, $\mathrm{Cr}$ 指红色色度分量。 人眼对亮度分量更敏感, 对色度分量不太敏感, 可以通过减少对色度分量采样来达到压缩图 像的目的。图 2 是 $\mathrm{YCbCr}$ 色彩空间示意图。

\begin{tabular}{|c|c|c|c|}
\hline Y0 & Y1 & Y2 & Y3 \\
\hline $\mathrm{Cb} 0$ & $\mathrm{Cb} 1$ & $\mathrm{Cb} 2$ & $\mathrm{Cb} 3$ \\
\hline $\mathrm{Cr} 0$ & $\mathrm{Cr} 1$ & $\mathrm{Cr} 2$ & $\mathrm{Cr} 3$ \\
\hline $\mathrm{Y} 4$ & $\mathrm{Y} 5$ & $\mathrm{Y} 6$ & $\mathrm{Y} 7$ \\
\hline $\mathrm{Cb} 4$ & $\mathrm{Cb} 5$ & $\mathrm{Cb} 6$ & $\mathrm{Cb} 7$ \\
\hline $\mathrm{Cr} 4$ & $\mathrm{Cr} 5$ & $\mathrm{Cr} 6$ & $\mathrm{Cr} 7$ \\
\hline
\end{tabular}

(a) $\mathrm{YCbCr} 4: 4: 4$

\begin{tabular}{|c|c|c|c|}
\hline YO & Y1 & Y2 & Y3 \\
\hline \multirow[t]{2}{*}{$\mathrm{CbO}$} & & $\mathrm{Cb} 2$ & \\
\hline & $\mathrm{Cr} 1$ & & $\mathrm{Cr} 3$ \\
\hline Y4 & Y5 & Y6 & Y7 \\
\hline \multirow[t]{2}{*}{$\mathrm{Cb} 4$} & & $\mathrm{Cb} 6$ & \\
\hline & Cr5 & & $\mathrm{Cr} 7$ \\
\hline
\end{tabular}

(b) $\mathrm{YCbCr} 4: 2: 2$

图 $2 \mathrm{YCbCr}$ 色彩空间示意图

对于拍摄的图像, 通常是 RGB 存储的, 对于阴影检测和去除的第一步, 需要将其转换为 $\mathrm{YCbCr}$ 色彩空间, RGB 转 $\mathrm{YCbCr}$ 公式如 (5) - (7) 所示。

$\mathrm{Y}^{\prime}=0.299 * \mathrm{R}^{\prime}+0.587 * \mathrm{G}^{\prime}+0.114 * \mathrm{~B}^{\prime}$

$\mathrm{Cb}^{\prime}=-0.147 * \mathrm{R}^{\prime}-0.289 * \mathrm{G}^{\prime}+0.436 * \mathrm{~B}^{\prime}=0.492 *\left(\mathrm{~B}^{\prime}-\mathrm{Y}^{\prime}\right)$

$\mathrm{Cr}^{\prime}=0.615 * \mathrm{R}^{\prime}-0.515^{*} \mathrm{G}^{\prime}-0.100 * \mathrm{~B}^{\prime}=0.877 *\left(\mathrm{R}^{\prime}-\mathrm{Y}^{\prime}\right)$

经参考文献可知, 亮度分量 $Y$ 部分特征更能表现一个阴影的特征（阴影往往要比非阴影区域 视觉表现上要暗), 所以在得到 $\mathrm{YCbCr}$ 之后, 需要计算 $\mathrm{Y}$ 分量的直方图, 并计算 $\mathrm{Y}$ 分量的像 素的整体平均值, 以此作为基底和图像中的其他像素进行对比, 并通过判决得到该像素点是 否是阴影, 如果是阴影的话, 计算整个阴影像素亮度的和, 并计算外部非阴影区域的像素的 亮度的和, 将阴影区域像素替换为计算的亮度模型的图像, 亮度模型 ${ }^{[8]}$ 可以用公式 (8) 来表 示。

$$
I^{\prime}=\frac{L+1}{S+1} I
$$

其中 I'为新的像素亮度值, $\mathrm{L}$ 为外部非阴影区域的像素的和, $\mathrm{S}$ 为阴影区域像素的和, I 为原 始图像的亮度值。整体流程图和效果图如图 3、图 4 所示。 


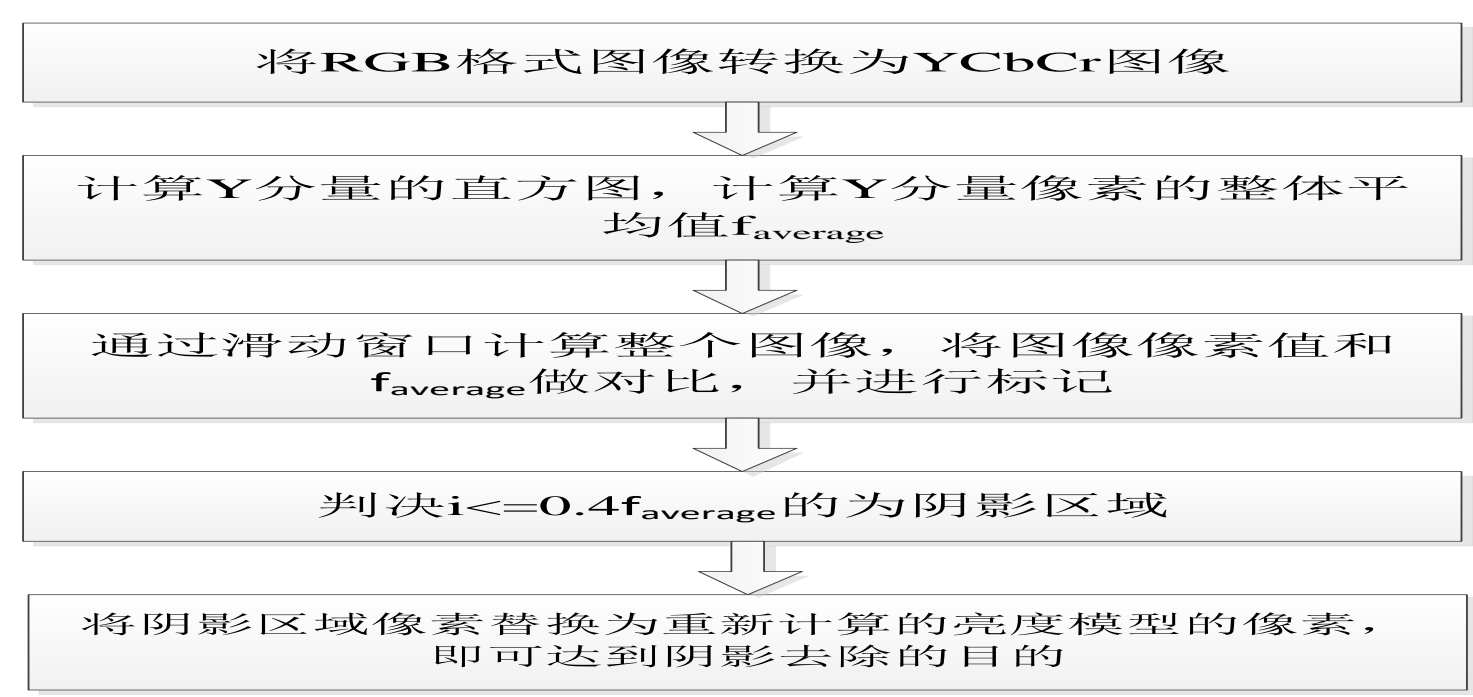

\section{图 $3 \mathrm{YCbCr}$ 阴影检测算法}

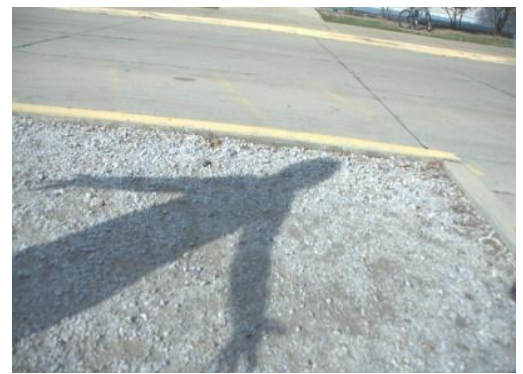

(a)原图

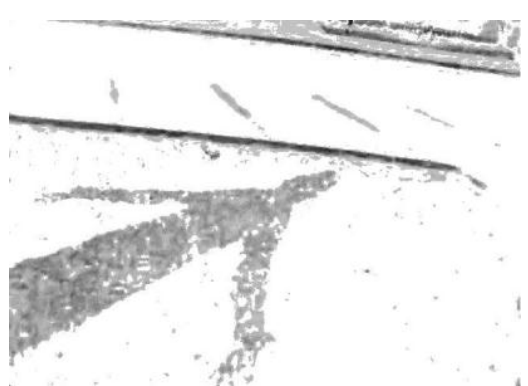

(b)阴影检测图像

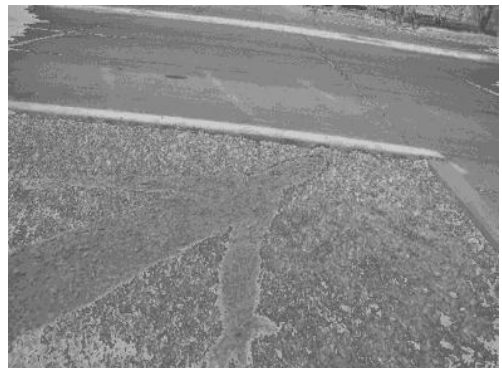

(c)阴影去除图像

图 4 阴影检测与阴影去除图像

\section{4. 结论}

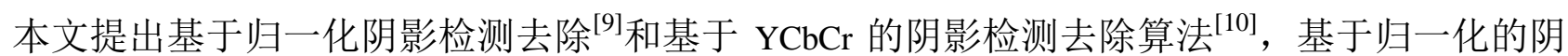
影检测和去除算法具有运算简单但对目标物体有比较严格的限制, 使用范围受到限制; 基于 $\mathrm{YCbCr}$ 的阴影检测去除算法对阴影的检测和去除有较好的效果, 但是也可以看到在阴影边缘 部分的痕迹没有去除干净, 存在残留现象, 需要后续进行调整。也可以看到要想开发出一种 效果较好、运算速度较快的阴影检测与去除算法, 还有较长的路要走。

\section{参考文献}

[1] 陈铁民, 李勇敢. 一种阴影图像边缘检测的新方法[J]. 电子技术应用, 2006, 32(9): 33-35.

[2] Leone A, Distante C. Shadow detection for moving objects based on texture analysis[J]. Pattern Recognition, 2007, 40(4):1222-1233.

[3] Guo R, Dai Q, Hoiem D. Paired Regions for Shadow Detection and Removal[J]. IEEE Transactions on Software Engineering, 2013, 35(12):2956-67.

[4] Osher S, Fedkiw R. Level set methods and dynamic implicit surfaces[M]. Springer Verlag, 2003.

[5] Visbal M R, Gaitonde D V. On the use of higher-order finite-difference schemes on curvilinear and deforming meshes [J]. Journal of Computational Physics, 2002, 181(1): 155-185. 
[6] Lankton S, Tannenbaum A. Localizing region-based active contours[J]. Image Processing, IEEE Transactions on, 2008, 17(11): 2029-2039.

[7] Adalsteinsson D. A fast level set method for propagating interfaces[D]. University of California, 1994.

[8] Guo R, Dai Q, Hoiem D. Single-image shadow detection and removal using paired regions [J]. Cvpr, 2011, 32(14):2033-2040.

[9] 王军利，王树根. 一种基于RGB彩色空间的影像阴影检测方法[J]. 信息技术, 2002(12):7-8.

[10] Wang J M, Chung Y C, Chang C L, et al. Shadow detection and removal for traffic images [J]. Networking Sensing \& Control IEEE International Conference on, 2004, 1:649-654 Vol.1.

\section{Acknowledgement}

This research was financially supported by the 2015 Research Project of Higher Education Reform in Henan Province (15B520036) and the 2016 Research Project of the Key Scientific and Technological in Henan Province (162102210119)

\section{References}

[1] Tiemin Chen, Yonggan Li. A New Method of Shadow Image Edge Detection [J]. Application of Electronic Technique, 2006, 32 (9): 33-35.

[2] Leone A, Distante C. Shadow detection for moving objects based on texture analysis[J]. Pattern Recognition, 2007, 40(4):1222-1233.

[3] Guo R, Dai Q, Hoiem D. Paired Regions for Shadow Detection and Removal[J]. IEEE Transactions on Software Engineering, 2013, 35(12):2956-67.

[4] Osher S, Fedkiw R. Level set methods and dynamic implicit surfaces [M]. Springer Verlag, 2003.

[5] Visbal M R, Gaitonde D V. On the use of higher-order finite-difference schemes on curvilinear and deforming meshes [J]. Journal of Computational Physics, 2002, 181(1): 155-185.

[6] Lankton S, Tannenbaum A. Localizing region-based active contours [J]. Image Processing, IEEE Transactions on, 2008, 17(11): 2029-2039.

[7] Adalsteinsson D. A fast level set method for propagating interfaces [D]. University of California, 1994.

[8] Guo R, Dai Q, Hoiem D. Single-image shadow detection and removal using paired regions [J]. Cvpr, 2011, 32(14):2033-2040.

[9] Wang Junli,Wang Shugen.A Shadow Detection Method of Color Image Based on RGB Color Space[J].INFORMATION TECHNOLOGY, 2002(12):7-8.

[10]Wang J M, Chung Y C, Chang C L, et al. Shadow detection and removal for traffic images [J]. Networking Sensing \& Control IEEE International Conference on, 2004, 1:649-654 Vol.1.

作者简介：第一作者范建德（1985-），性别男，籍贯河南南阳，职称讲师，主要研究方向为 机器视觉, E-mail: 568498042@qq.com. 